\title{
Focus on Endovascular Solutions for Cerebrovascular Diseases: Report of the Fourth International Stroke Summit, Nanjing, China, July 25-27, 2008
}

\author{
Gelin Xu Songming Cheng Xinfeng Liu \\ Department of Neurology, Jinling Hospital, Nanjing University School of Medicine, Nanjing, China
}

Stroke is one of the leading causes of death and long-term disability worldwide [1]. The continued aging of the global population in recent years is associated with an increase in stroke prevalence, especially in developing countries. As a result, the socioeconomic cost associated with stroke is climbing, the disability-adjusted life-years lost to stroke are increasing, and these trends will probably become stronger over the next 20 years [2]. In striking contrast to the persistently increasing number of patients, efficacious therapies for stroke are still very limited. Therefore, exploring novel strategies for stroke prevention and treatment is of utmost importance.

During the past two decades, endovascular interventions have been one approach to stroke prevention and treatment. Endovascular techniques were originally developed for the peripheral vasculature and have seen much success in coronary vasculature surgery. The techniques were introduced to cerebral vasculature surgery in the early 1980s for treating carotid stenosis [3]. Several other neuroendovascular strategies have been developed since then, including angioplasty and stenting for cerebral artery stenosis, coil embolization for intracranial aneurysm and mechanical embolus retrieval for acute intra-artery thrombosis, which all have inspired keen research interest.

The International Stroke Summit is a regular regional conference endorsed by the World Stroke Organization (WSO) for promoting stroke research and management in developing countries. This annual conference has been successfully conducted 4 times by now. According to the organizational plan, the Scientific Committee of the conference suggests a main topic each year. The first summit was held in Beijing in September 2004, with more than 2,000 researchers and doctors from China and other countries. Epidemiology and prevention of stroke was the main topic for the Beijing meeting [4]. The second summit was held in Nanjing in August 2006, with in-hospital management of acute stroke as the main topic [5]. The third summit took place in Wuhan in November 2007 and had pre-hospital management and post-hospital rehabilitation of stroke patients as its main topic $[6,7]$.

\section{KARGER}

Fax +41613061234 E-Mail karger@karger.ch www.karger.com
(C) 2008 S. Karger AG, Basel

1015-9770/08/0266-0667\$24.50/0

Accessible online at:

www.karger.com/ced
The Fourth International Stroke Summit was held on July 2527, 2008, also in Nanjing. In view of the significant progress in neuroendovascular techniques in recent years, the recommended main conference topic for this year was endovascular solutions for stroke prevention and treatment [8]. As in the previous meetings, the aim of narrowing the gap between developing and developed countries in stroke management dominated the agenda of the conference. Besides endovascular interventions for stroke, several other topics - acute thrombolysis after cerebral infarction, pre-hospital management of stroke, prevention and treatment of vascular cognitive impairment - also garnered much interest.

The Fourth International Stroke Summit was co-chaired by Xinfeng Liu from Nanjing University, China, Werner Hacke from the University of Heidelberg, Germany, and Michael Chopp from the Henry Ford Hospital in Detroit, USA. In his opening address, Werner Hacke delivered the very best wishes of Geoffrey Donnan, president of the WSO. Professor Hacke then briefly spoke about the events leading up to the founding and the ongoing development of this conference during the past 4 years. He encouraged Chinese doctors and researchers to participate in more international stroke conferences and to publish their research results in outstanding stroke journals, such as Stroke and Cerebrovascular Diseases.

Xinfeng Liu opened his lecture with a brief introduction to endovascular medicine. He then shifted his topic to the recent progress in endovascular techniques used for the treatment of atherosclerotic diseases. He addressed the unique status that carotid angioplasty and stenting (CAS) enjoys in China as well as in many other developing countries. Due to a shortage of skilled neurosurgeons and the reluctance of patients to undergo a major operation in the neck, carotid endarterectomy (CEA) is not available in most Chinese hospitals. Given this situation, most Chinese patients with carotid stenosis cannot be treated according to the guidelines tailored for European and North American populations, in which CEA is the first choice for severe carotid stenosis and only patients considered high risk for surgery are recommended to have CAS. Since CEA is a widely practiced operation 
with proven efficacy, almost all clinical trials evaluating CAS feasibility were designed to compare it with CEA. As a result, no randomized clinical trial directly comparing CAS and medicinal treatment is available to date. Liu pointed out that CAS is developing very fast in China and patient compliance for this minimally invasive procedure is very encouraging. But to improve outcomes of cerebrovascular diseases in Chinese patients, a multi-center randomized clinical trial to confirm the feasibility of CAS in patients for whom CEA is not available is absolutely essential.

Werner Hacke delivered a lecture on the management of severe stroke. He emphasized that penumbra is a very individualized concept: there is no uniform pattern of penumbra and hence, there is no narrowly defined time window in treating individual patients. The reason for a 3-hour time window is simply that, by this time, the majority of stroke victims will have some penumbral tissue and remaining vessel occlusion. Since penumbral tissue might die over time, one should act quickly. After 3-4.5 h, there may still be a reasonable number of patients with some significant degree of salvable penumbra. After this time, some patients still survive, but the number gets smaller by the hour. Based on the individualized concept of penumbra, Hacke then discussed the rationales and indications of three major strategies for severe stroke: rtPA thrombolysis, decompressive surgery and hypothermia. He also introduced sonolysis with microbubbles and mechanical retrieval devices which he believes can increase the recanalization rates in thrombolytic patients.

Michael Chopp from Detroit made a speech on stimulating brain plasticity and recovery after stroke. He introduced his new hypothesis of brain remodeling after stroke by combining strategies of angiogenesis, synaptogenesis and neurogenesis. Results from his series studies indicated that clotting in the middle cerebral artery can activate integrins and matrix metalloproteinases, which may trigger a cascade of neurochemical events and finally result in permanent vascular and neuronal damages. Because activation of the GPIIa/IIIb receptor plays a key role in the process of thrombosis, simultaneous intra-arterial administration of thrombolytic agents and abciximab may enhance vascular patency.

Zhenguo Liu, a cardiologist from Ohio University, USA, discussed patent foramen ovale (PFO) as a potential risk for cryptogenic stroke and also introduced several strategies for treating PFO. Currently, PFOs are treated variably with antiplatelet agents, anticoagulants, surgical closure, or, increasingly, endovascular closure devices. Liu emphasized the importance of a randomized clinical trial to clarify whether the risks associated with invasive endovascular device placement are outweighed by a long-term reduction in recurrent vascular events.
Several speakers discussed the feasibility of surgical and endovascular treatments for intracranial aneurysms. Jianmin Liu, a neurosurgeon from Shanghai, reported his experience in treating unruptured complex intracranial aneurysm with a combination of coiling embolization and stenting. Results from his study indicate that this combined endovascular strategy has competitive short-term effects compared to open skull surgery.

Although neuroendovascular techniques have received popularity in China in recent years, a feasible examination and accreditation system for neuroendovascular training has not been established. Only a few programs have framed a standard training protocol and no qualifying examinations for interventional neurology are available. Because the practitioners are from different specialties and with widely different backgrounds, it is very important to organize an accreditation board that would include experts from different specialties. Wei $\mathrm{Wu}$, a neurologist who has been trained as an interventionist recently, discussed the current status of endovascular training in China. Wu also mentioned the learning curve in endovascular training. He said that a novice should have performed at least 100 cases of angiography before performing his first CAS procedure.

Although having been discussed in the previous conference, pre-hospital management of stroke was still a hot topic in this conference. It has been reported that Chinese patients have a longer pre-hospital delay after stroke onset than western patients. Poor transportation and ignorance of stroke warning signs are among the main causes for this disadvantage. Therefore, some speakers called for a reform of the ambulance service and insisted that patient education is the first step to improve stroke outcomes.

Jianping Jia from Beijing reported his study concerning the status of vascular cognitive impairment. His results show that vascular cognitive impairment is more prevalent in Chinese stroke patients than in western patients, which he contributes to the high ratio of uncontrolled modifiable risk factors for cerebrovascular diseases and dementia. Thirty selected abstracts submitted to the conference were published in Cerebrovascular Diseases [8].

About 830 doctors and physicians from China and other countries participated in the meeting. In the closing ceremony, the organizers announced that the next meeting, the Fifth International Stroke Summit, will be held on July 17-20, 2009, in Nanjing, China. They encouraged researchers and clinicians around the world to join the next meeting and continue to contribute to the communication between East and West. Detailed information concerning the conference is now available at www.stroke. net.cn.

\section{References}

-1 Donnan GA, Fisher M, Macleod M, Davis SM: Stroke. Lancet 2008;371:1612-1623.

$\checkmark 2$ Lopez AD, Mathers CD, Ezzati M, Jamison DT, Murray CJ: Global and regional burden of disease and risk factors, 2001: systematic analysis of population health data. Lancet 2006;367:1747-1757.

$\checkmark 3$ Yadav JS: Management practices in carotid stenting. Cerebrovasc Dis 2001;11(suppl 2):18-22.
4 ISS Regional Cerebrovascular Diseases Conference (abstracts). Cerebrovasc Dis 2004; 18: 352-378. www.karger.com/doi/10.1159/ 000081968 .

5 Second International Stroke Summit (abstracts). Cerebrovasc Dis 2006;22:318-329. www.karger.com/doi/10.1159/000094832.

6 Third International Stroke Summit (abstracts). Cerebrovasc Dis 2007;24:483-494. www.karger.com/doi/10.1159/000109749.
$7 \mathrm{Xu}$ G: Building a platform for East-West communication in stroke research: report of the Third International Stroke Summit, Wuhan, China, November 1-3, 2007. Cerebrovasc Dis 2008;25:279-280.

8 Liu X, Pu C, Hacke W, Chopp M, Zhang S, $\mathrm{Xu}$ G: Fourth International Stroke Summit. Nanjing, China, July 25-27, 2008. Cerebrovasc Dis $2008 ; 26: 212-222$. 